\title{
Long Noncoding RNA NEAT1 Aggravates A $\beta$-Induced Neuronal Damage by Targeting miR-107 in Alzheimer's Disease
}

\author{
Sha $\mathrm{Ke}^{1}$, Zhaohui Yang ${ }^{2}$, Fei Yang ${ }^{1}$, Xiaoming Wang ${ }^{1}$, Juan Tan ${ }^{1}$, and Bo Liao ${ }^{3}$ \\ Departments of ${ }^{1}$ Neurology, ${ }^{2}$ Burn and Plastic Surgery, and ${ }^{3}$ Urology, Affiliated Hospital of North Sichuan Medical College, Nanchong, Sichuan, \\ China.
}

\begin{abstract}
Purpose: Alzheimer's disease (AD) is the most common neurodegenerative disease, with a rising prevalence worldwide. Long noncoding RNAs (IncRNAs) have been found to play important roles in the development and treatment of AD. However, the exact role of IncRNA nuclear enriched abundant transcript 1 (NEAT1) in neuronal damage in AD is largely unknown.

Materials and Methods: The AD model was established in SH-SY5Y and SK-N-SH cells via treatment with amyloid $\beta_{1-42}(\mathrm{~A} \beta)$. The expression of NEAT1 and microRNA-107 (miR-107) was measured by quantitative real-time polymerase chain reaction. Cell viability and apoptosis were detected by MTT assay, immunocytochemistry, and flow cytometry. The expression of phosphorylated tau protein (p-Tau) was measured by Western blot. The interaction between NEAT1 and miR-107 was explored by bioinformatics analysis, luciferase activity, and RNA immunoprecipitation assays.

Results: NEAT1 expression was enhanced in A $\beta$-treated SH-SY5Y and SK-N-SH cells, and its knockdown attenuated A $\beta$-induced inhibition of viability and promotion of apoptosis and p-Tau levels. NEAT1 was indicated as a decoy of miR-107. miR-107 abundance was reduced in A $\beta$-treated cells, and its overexpression reversed A $\beta$-induced injury. Moreover, interference of miR-107 abated silencing of NEAT1-mediated inhibition of neuronal damage in A $\beta$-treated SH-SY5Y and SK-N-SH cells.

Conclusion: LncRNA NEAT1 aggravated A $\beta$-induced neuronal damage by sponging miR-107, indicating a novel avenue for treatment of $\mathrm{AD}$.
\end{abstract}

Key Words: Alzheimer's disease, NEAT1, miR-107, neuronal damage

\section{INTRODUCTION}

Alzheimer's disease (AD), as a major cause of neurodegenerative disease, poses a major healthcare challenges to older adults worldwide. ${ }^{1}$ The pathology of $\mathrm{AD}$ is characterized by cognitive loss and pathological hallmarks of amyloid and neurofibrillary tangles. ${ }^{2}$ Meanwhile, research has shown that amyloid $\beta$ pep-

Received: December 26, 2018 Revised: April 8, 2019

Accepted: April 30, 2019

Corresponding author: Bo Liao, MM, Department of Urology, Affiliated Hospital of North Sichuan Medical College, Wenhua road 63, Shunqing District, Nanchong 637000, Sichuan, China.

Tel: 86-0817-2262110, Fax: 86-0817-2262110, E-mail: liaobonsmc@163.com

-The authors have no potential conflicts of interest to disclose.

(C) Copyright: Yonsei University College of Medicine 2019

This is an Open Access article distributed under the terms of the Creative Commons Attribution Non-Commercial License (https://creativecommons.org/licenses/ by-nc/4.0) which permits unrestricted non-commercial use, distribution, and reproduction in any medium, provided the original work is properly cited. tides $\left(A \beta_{1-40}\right.$ and $\left.A \beta_{1-42}\right)$ and hyperphosphorylation of tau protein (p-Tau) contribute to AD development. ${ }^{3,4}$ Although great attention has been given to the diagnosis and treatment of $\mathrm{AD}$, strategies for preventing $\mathrm{AD}$ progression remain limited.

Noncoding RNAs (ncRNAs), including long ncRNAs (lncRNAs) and microRNAs (miRNAs), have been implicated in the onset and pathogenesis of AD. ${ }^{5}$ Emerging evidence suggests lncRNAs as promising targets in the treatment, diagnosis, and prevention of neurodegenerative diseases, including AD. ${ }^{6}$ For example, lncRNA sex-determining region $\mathrm{Y}$ (SRY)-related $\mathrm{HMG}$ box (SOX) 21 antisense RNA 1 (SOX21-AS1) knockdown attenuated neuronal oxidative injury in mice with $\mathrm{AD}$ by regulating Wnt signaling via Frizzled 3/5 (FZD3/5). ${ }^{7}$ LncRNA early B cell factor 3 antisense RNA (EBF3-AS) facilitated neuronal apoptosis in an in vitro AD model. ${ }^{8}$ LncRNA nuclear enriched abundant transcript 1 (NEAT1) was indicated as a promising target in neurodegenerative diseases. Chanda, et al. ${ }^{9}$ reported that NEAT1 is upregulated in Huntington's disease and that its 
knockdown weakens the formation of aggregates. Furthermore, NEAT1 has been described as promoting neurotoxin 1-methyl-4-phenyl-1,2,3,6-tetrahydropyridine (MPTP)-induced autophagy by regulating phosphatase and tensin homolog deleted on chromosome ten-induced kinase 1 (PINK1) in Parkinson's disease. ${ }^{10}$ Additionally, NEAT1 knockdown has been found to increase cell viability and to suppress apoptosis in a MPTP/MPP ${ }^{+}$-induced Parkinson's disease model, ${ }^{11}$ and research has indicated that NEAT1 is highly expressed in the temporal cortex and hippocampus of $\mathrm{AD}$ patients. ${ }^{12}$ However, the potential role of NEAT1 in AD progression and its underlying mechanism are largely unclear.

miRNAs are a class of small ncRNAs, and have been described as promising diagnostic and therapeutic tools for $\mathrm{AD}$ treatment. ${ }^{13}$ miR-107 has been shown to be associated with pathogenesis in human diseases. ${ }^{14-16}$ Moreover, miR-107 is reported to be downregulated in $\mathrm{AD}$ and to play an essential role in $\mathrm{AD}$ pathology. ${ }^{17}$ Bioinformatics analysis has predicted the potential binding sites of NEAT1 and miR-107, indicating a potential interaction between them. Therefore, we hypothesized that miR-107 might be involved in NEAT1-mediated progression of $\mathrm{AD}$. In this study, we established an AD model using SH-SY5Y and SK-N-SH cells treated with amyloid $\beta_{1-42}(A \beta)$. Therein, we explored the effect of NEATl on A $\beta$-induced neuronal damage and its underlying mechanism.

\section{MATERIALS AND METHODS}

\section{Cell culture and treatment}

The human neuroblastoma cell lines (SH-SY5Y and SK-N-SH) and human embryonic kidney cells 293T were purchased from American Tissue Culture Collection (ATCC; Manassas, VA, USA). All cells were maintained in Dulbecco's Modified Eagle Medium (Gibco, Carlsbad, CA, USA) with $10 \%$ fetal bovine serum (Gibco), $100 \mathrm{U} / \mathrm{mL}$ of penicillin, and $100 \mu \mathrm{g} / \mathrm{mL}$ of streptomycin (Gibco) at $37^{\circ} \mathrm{C}$ and $5 \% \mathrm{CO}_{2}$. For establishment of $\mathrm{AD}$ model in vitro, SH-SY5Y and SK-N-SH cells were treated with different concentrations $(0,5,10$, or $20 \mu \mathrm{M})$ of $\mathrm{A} \beta$ (purity: 95.64\%; MedChemExpress, Monmouth, NJ, USA) in dimethyl sulfoxide (DMSO; Thermo Fisher, Wilmington, DE, USA) for $24 \mathrm{~h}$ or $10 \mu \mathrm{M} \mathrm{A} \beta$ for different treatment times $(0,12,24$, or $48 \mathrm{~h})$.

Small interfering RNA (siRNA) against NEAT1 (si-NEAT1) (5'-GUGAGAAGUUGCUUAGAAACUUUCC-3'), siRNA negative control (si-NC) (5'-UUCUCCGAACGUGUCACGUTT-3'), NEAT1 overexpression vector (NEAT1) (Forward, 5'-CTTC CTCССТTTAACTTATCCATTCAC-3'; Reverse, 5'-CTCTTCC TCCACCATTACCAACAATAC-3'), pcDNA empty vector (pcDNA), miR-107 mimic (miR-107) (Forward, 5'-AGCAGCAUUG UACAGGGCUAUCA-3'; Reverse, 3'-AUAGCCCUGUACAAU GCUGCUUU-5'), mimic negative control (miR-NC) (Forward, 5'-UUCUCCGAACGUGUCACGUTT-3'; Reverse, 3'-ACGUGA CACGUUCGGAGAATT-5'), miR-107 inhibitor (anti-miR-107)
(5'-UGAUAGCCCUGUACAAUGCUGCU-3'), and inhibitor negative control (anti-miR-NC) (5'-CAGUACUUUUGUGUA GUACA-3') were synthesized by Genepharma (Shanghai, China). Cell transfection was conducted in SH-SY5Y and SK-N-SH cells for $48 \mathrm{~h}$ using the Lipofectamine 2000 (Invitrogen, Carlsbad, CA, USA) platform according to the manufacturer's instructions prior to $A \beta$ treatment.

\section{Quantitative real-time polymerase chain reaction} Total RNA extracted from cells using TRIzol reagent (Invitrogen) was used for cDNA synthesis by TransScript miRNA Firststand cDNA Synthesis SuperMix (TransGen Biotech, Beijing, China) according to the manufacturer's instructions. Subsequently, cDNA was diluted and used for quantitative real-time polymerase chain reaction (qRT-PCR) with SYBR green (Applied Biosystems, Foster City, CA, USA) using the ABI 7300 system (Applied Biosystems). The relative expressions of NEAT1 and miR-107 were measured with GAPDH or U6 small RNA as internal control using $2^{-\Delta \Delta C t}$ method. ${ }^{18}$ The primers used in this study were as follows: NEAT1 (Forward, 5'-TGGCTAGCTCAG GGCTTCAG-3'; Reverse, 5'-TCTCCTTGCCAAGCTTCCTTC-3'), miR-107 (Forward, 5'-AGCAG CATTGTACAGGG-3'; Reverse, 5'-GTGCAGGGTCCGAGGT-3'), U6 (Forward, 5'-CTCGCTTC GGCAGCACA-3'; Reverse, 5'-AACGCTTCACGAATTTGCGT-3'), GAPDH (Forward, 5'-TATGATGATATCAAGAGGGTAGT-3'; Reverse, 5'-TGTATCCAAACTCATTGTCATAC-3').

\section{Cell viability}

MTT (3-(4,5-dimethyl-2-thiazolyl)-2,5-diphenyl-2-H-tetrazolium bromide) assay was conducted to measure cell viability. SH-SY5Y and SK-N-SH cells $\left(1 \times 10^{4}\right.$ cells per well $)$ were seeded into 96-well plates and treated with $10 \mu \mathrm{M} \mathrm{A} \beta$ for $24 \mathrm{~h}$. Then cells were incubated with $0.5 \mathrm{mg} / \mathrm{mL}$ of MTT solution (Sigma, St. Louis, MO, USA) for another $4 \mathrm{~h}$. Subsequently, $100 \mu \mathrm{L}$ of DMSO was added to each well until the solubilization of formazan. Absorbance was measured at $490 \mathrm{~nm}$ using a microplate reader (Bio-Rad, Hercules, CA, USA). All samples were prepared in triplicate, and relative cell viability was normalized to non$\mathrm{A} \beta$ group.

\section{Immunocytochemistry}

SH-SY5Y and SK-N-SH cells transfected with si-NEAT1 or si$\mathrm{NC}$ were cultured in 24-well plates and exposed to $10 \mu \mathrm{M} \mathrm{A} \beta$ for $24 \mathrm{~h}$. Cells were then fixed with $4 \%$ paraformaldehyde and permeabilized with $0.1 \%$ Triton (Sigma). After blocked with $1 \%$ bovine serum albumin (Sigma), cells were incubated with Alexa Flour 488-conjugated primary antibodies against Ki67 (ab197234, Abcam, Cambridge, MA, USA). A DAPI solution (Sigma) was used for nuclear staining (blue).

\section{Cell apoptosis}

Cell apoptosis was measured using Annexin V-FITC/PI apoptosis detection kits (Solarbio, Beijing, China) via flow cytome- 
try according to the manufacturer's instructions. After washing with PBS, SH-SY5Y and SK-N-SH cells were resuspended in binding buffer and then stained with $5 \mu \mathrm{L}$ of Annexin VFITC for $10 \mathrm{~min}$ and $5 \mu \mathrm{L}$ of PI for 5 min in the dark at room temperature. Stained cells were analyzed using a flow cytometer (Becton Dickinson, San Jose, CA, USA). Samples from each group were prepared in triplicate, and experiments were repeated three times. The apoptotic rate comprised the percentage of cells in early apoptosis and last apoptosis or death.

\section{Western blot}

SH-SY5Y and SK-N-SH cells were washed with cold PBS and then lysed with RIPA lysis buffer (Beyotime Biotech, Shanghai, China). After centrifugation at $12000 \times$ g for $5 \mathrm{~min}$, total proteins in supernatant were quantified using BCA protein assay kits (Thermo Fisher). Equal amounts of protein $(25 \mu \mathrm{g})$ were denatured in SDS-PAGE sample loading buffer (Beyotime Biotech) at $100^{\circ} \mathrm{C}$ for $5 \mathrm{~min}$ and then separated on SDS-PAGE gel. Polyvinylidene difluoride membranes (Millipore, Billerica, MA, USA) were used for transfer of protein and were blocked with $5 \%$ non-fat milk for $1 \mathrm{~h}$ at room temperature. Subsequently, the membranes were incubated with primary antibodies against p-Tau (ser396) (ab109390; Abcam) or $\beta$-actin (ab8227; Abcam) overnight at $4^{\circ} \mathrm{C}$ and then interacted with horseradish peroxidase-conjugated secondary antibody (ab6721; Abcam) for $2 \mathrm{~h}$ at room temperature. Protein signaling was visualized using enhanced chemiluminescence chromogenic substrate (Beyotime Biotech) and was analyzed with $\beta$-actin as a loading control.

\section{Bioinformatics analysis and luciferase activity assay} The putative binding sites of miR-107 and NEAT1 were predicted by bioinformatics analysis using StarBase online (http:// starbase.sysu.edu.cn/). The 3'-UTR sequences of NEAT1 carrying wild-type (WT) or mutant (MUT) putative binding sites of miR-107 were amplified and cloned into pmirGLO vectors (Promega, Madison, WI, USA) to synthesize luciferase reporter vectors (NEAT1-WT or NEAT1-MUT). 293T cells were cotransfected with NEAT1-WT or NEAT1-MUT and miR-107 or miR-NC using Lipofectamine 2000 according to the manufac-
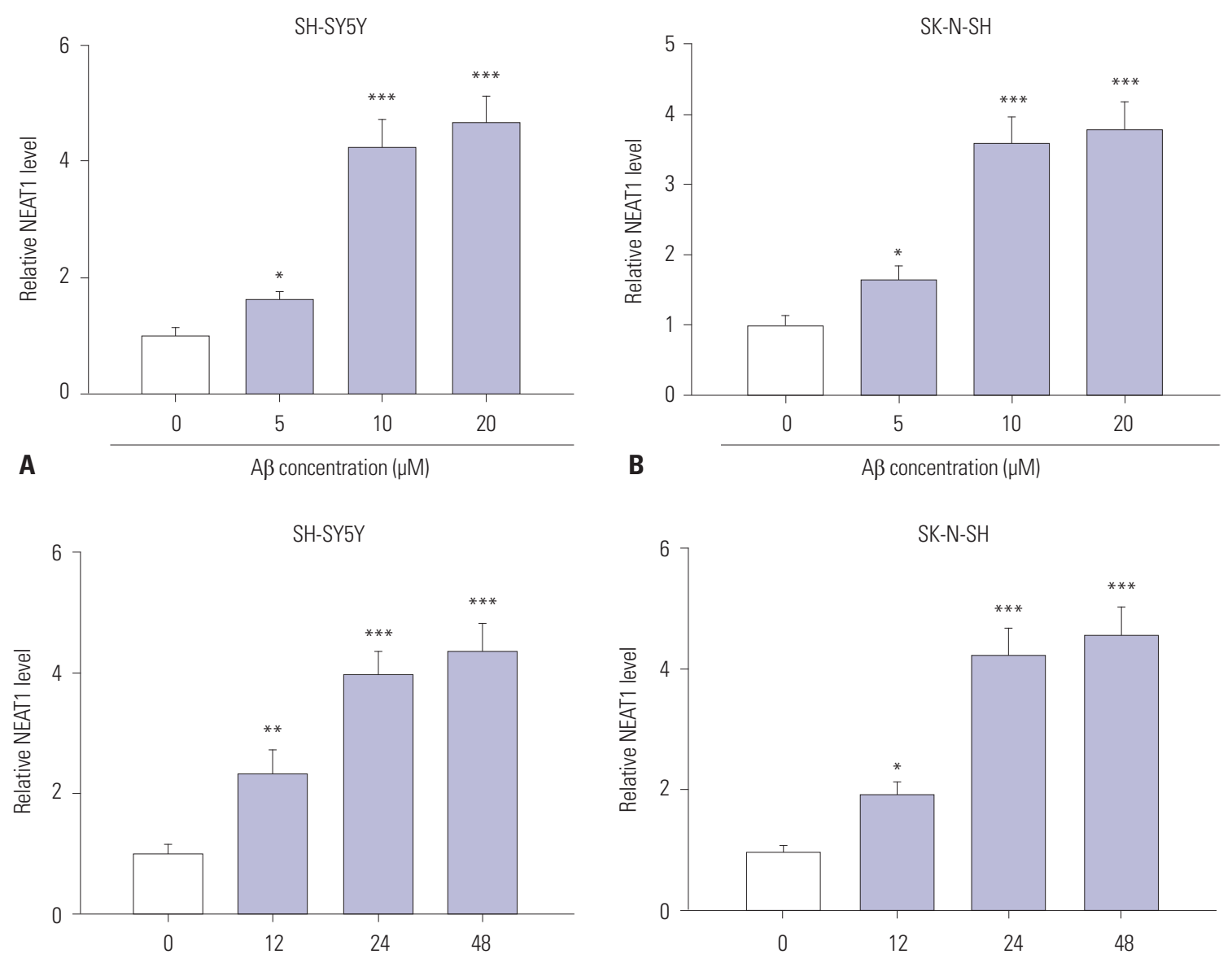

C

$\mathrm{A} \beta(10 \mu \mathrm{M})$ treatment time (hr)

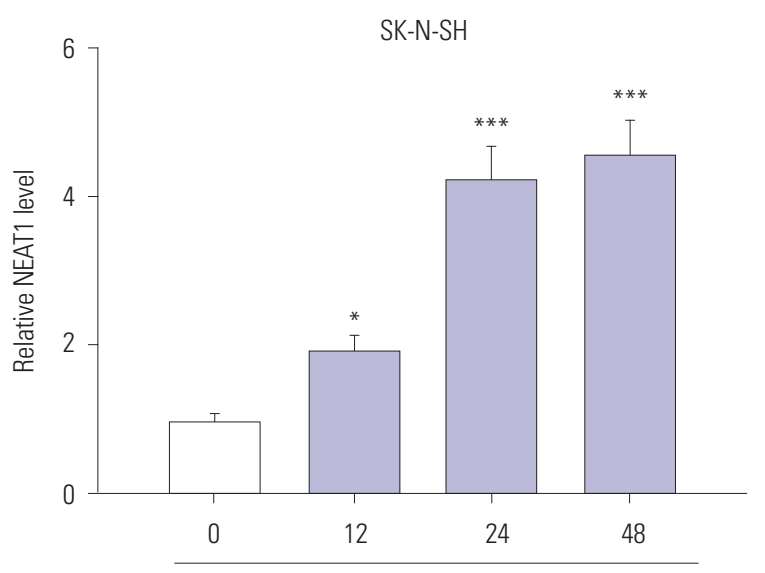

D

$A \beta(10 \mu M)$ treatment time $(h r)$

Fig. 1. Expression of NEAT1 is elevated in A $\beta$-treated SH-SY5Y and SK-N-SH cells. (A and B) The expression of NEAT1 was measured in SH-SY5Y and SK-N-SH cells after treatment of different concentrations $(0,5,10$, and $20 \mu \mathrm{M})$ of $A \beta$ for $24 \mathrm{~h}$ by qRT-PCR. (C and D) The levels of NEAT1 were detected in SH-SY5Y and SK-N-SH cells after treatment of $10 \mu \mathrm{M}$ A $\beta$ for different treatment times $(0,12,24$, and $48 \mathrm{~h})$ by qRT-PCR. ${ }^{*} p<0.05$, ${ }^{* *} p<0.01$, ${ }^{* * *} p<0.001$. NEAT1, nuclear enriched abundant transcript 1; $A \beta$, amyloid $\beta_{1-42}$. 
turer's protocols. After transfection for $48 \mathrm{~h}$, cells were collected and luciferase activity was analyzed with luciferase assay kits (Promega) according to the manufacturer's instructions.

\section{RNA immunoprecipitation}

RNA immunoprecipitation (RIP) assay was conducted using Magna RNA immunoprecipitation kits (Millipore) according to the manufacturer's protocols. In brief, SH-SY5Y and SK-NSH cells transfected with miR-107 or miR-NC were lysed in RIP immunoprecipitation buffer containing magnetic beads bound with antibody against Ago2 or IgG. The enrichment of NEAT1 on beads was measured by qRT-PCR.

\section{Statistical analysis}

Data are presented as means \pm standard deviations from three independent experiments. Differences between groups were measured by one-way analysis of variance using GraphPad Prism 7 Software (GraphPad Inc., La Jolla, CA, USA). $p<0.05$ was regarded as statistically significant.

\section{RESULTS}

\section{NEAT1 expression is enhanced in A $\beta$-treated SH-SY5Y and SK-N-SH cells}

To explore the potential role of NEAT1 in AD progression, the
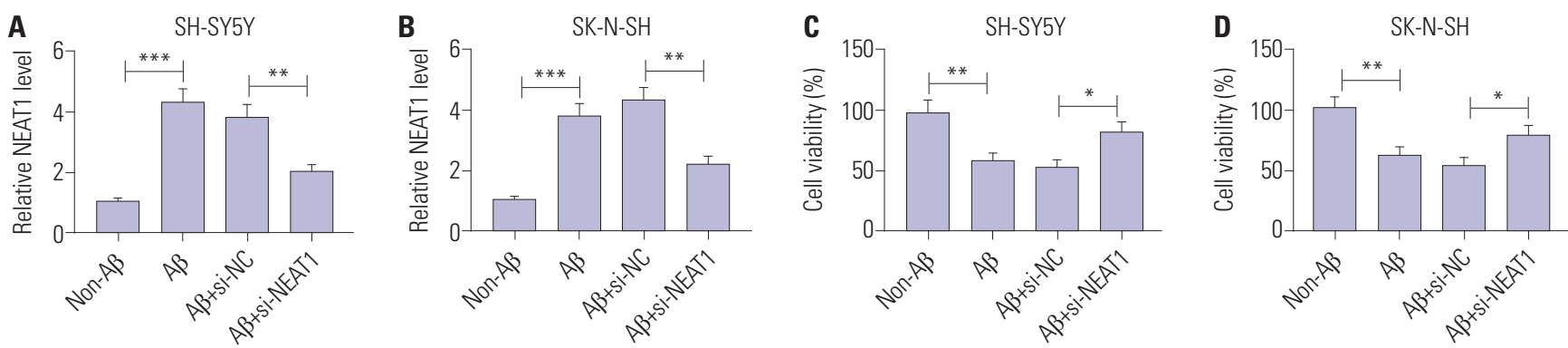

E

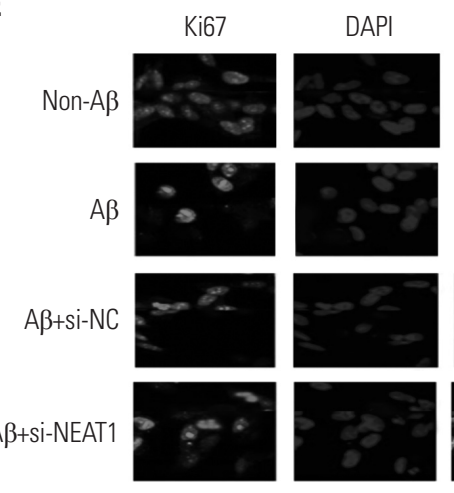

Merge
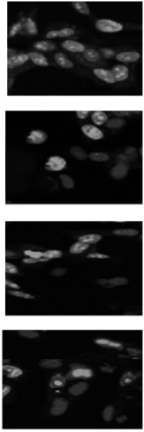

$\mathbf{F}$ SK-N-SH
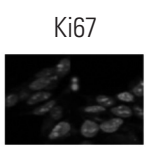
DAPI

Non-A $\beta$

A $\beta$
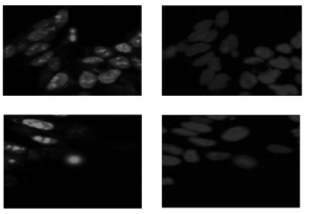

Merge
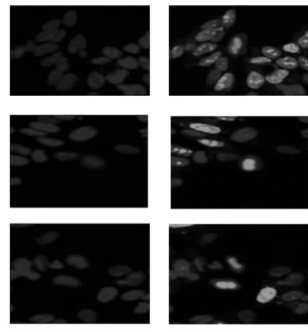

$A \beta+$ Si-NC
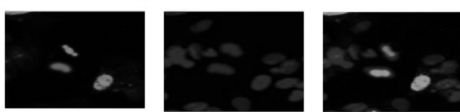

A $\beta+$ Si-NEAT1
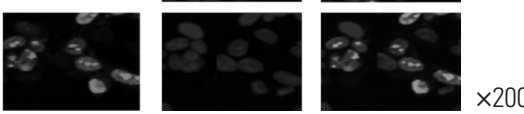

H

G

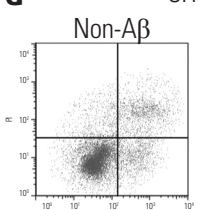

$A \beta+S i-N C$

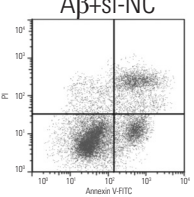

SH-SY5Y
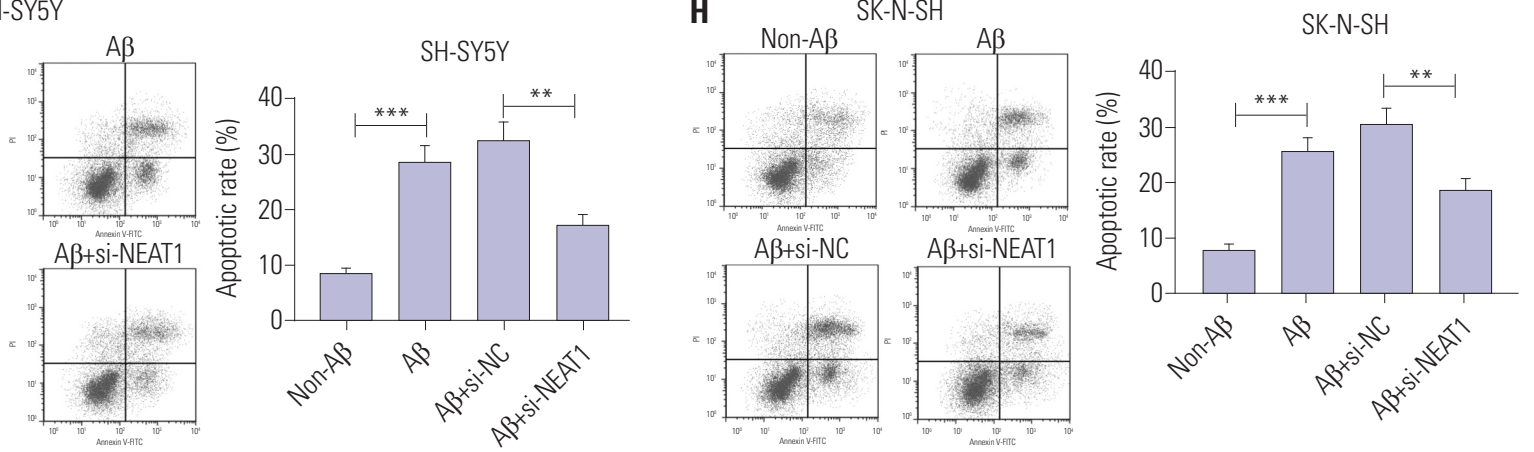

Fig. 2. Knockdown of NEAT1 attenuates A $\beta$-induced neuronal damage in SH-SY5Y and SK-N-SH cells. (A and B) The expression of NEAT1 was measured in SH-SY5Y and SK-N-SH cells transfected with si-NEAT1 or si-NC after treatment of $10 \mu \mathrm{M}$ A $\beta$ for $24 \mathrm{~h}$ by qRT-PCR. Cell viability (C and D), Ki67 expression ( $E$ and $F)$, and apoptosis ( $\mathrm{G}$ and $\mathrm{H}$ ) were detected in SH-SY5Y and SK-N-SH cells transfected with si-NEAT1 or si-NC after treatment of 10 $\mu \mathrm{M}$ A $\beta$ for $24 \mathrm{~h}$ by MTT, immunocytochemistry, and flow cytometry. ${ }^{*} p<0.05,{ }^{* *} p<0.01$, ${ }^{* * *} p<0.001$. NEAT1, nuclear enriched abundant transcript 1 ; A $\beta$, amyloid $\beta_{1-42 .}$ 
expression of NEAT1 was measured in an A $\beta$-induced AD model in vitro. As shown in Fig. $1 A$ and $B$, after treatment of $A \beta$ for $24 \mathrm{~h}$, NEAT1 levels significantly increased in SH-SY5Y and SK$\mathrm{N}$-SH cells in a concentration dependent manner, compared with those in the non-treated group. Moreover, an abundance of NEAT1 was obvious in $10 \mu \mathrm{M}$ A $\beta$-treated SH-SY5Y and SK$\mathrm{N}$-SH cells in a time dependent manner, compared with the non-treated group (Fig. 1C and D). SH-SY5Y and SK-N-SH cells treated with $10 \mu \mathrm{M} \mathrm{A} \beta$ for $24 \mathrm{~h}$ were used for the following experiments because of the significant alteration of NEAT1 levels.

\section{Knockdown of NEAT1 attenuates A $\beta$-induced neuro- nal damage in SH-SY5Y and SK-N-SH cells}

To investigate the effect of NEAT1 on AD progression, SH-SY5Y and SK-N-SH cells were transfected with si-NEAT1 or si-NC and then treated with $10 \mu \mathrm{M} \mathrm{A} \beta$ for $24 \mathrm{~h}$. As displayed in Fig. $2 \mathrm{~A}$ and $\mathrm{B}$, the abundance of NEAT1 was effectively reduced in SH-SY5Y and SK-N-SH cells upon transfection of si-NEAT1, compared with the si-NC group, after treatment of A $\beta$. MTT assay showed that cell viability was markedly inhibited by treatment of $\mathrm{A} \beta$ in SH-SY5Y and SK-N-SH cells, which was reversed by depletion of NEAT1 (Fig. 2C and D). Meanwhile, immunocytochemistry analysis revealed that exposure of $\mathrm{A} \beta$ significantly decreased Ki67 expression, and this effect was weakened via silencing NEAT1 (Fig. 2E and F). Furthermore, exposure of $\mathrm{A} \beta$ led to a strong increase in apoptosis of SH-SY5Y and SK-NSH cells, which was attenuated via down-regulation of NEAT1
(Fig. 2G and H). Furthermore, the effect of NEAT1 on accumulation of p-Tau was evaluated in A $\beta$-treated SH-SY5Y and SK-NSH cells. Western blot analysis demonstrated that protein levels of p-Tau were obviously increased in SH-SY5Y and SK-N-SH cells after treatment of $A \beta$, compared with the non-treated group, while it was greatly abated by inhibition of NEAT1 (Fig. 3). These findings indicated that $A \beta$ successfully induced neuronal damage, which was attenuated by NEAT1 silencing.

\section{MiR-107 is bound to NEAT1}

In order to elucidate the underlying mechanism accounting for NEAT1 involvement in AD development, we explored miRNA that bind to NEAT1 by bioinformatics analysis. StarBase online assay provided the putative binding sites of miR107 and NEAT1, suggesting that miR-107 might be targeted by NEAT1 (Fig. 4A). To validate the prediction, luciferase activity and RIP assays were conducted. Luciferase activity was obviously inhibited in $293 \mathrm{~T}$ cells transfected with miR-107, compared with that in cells treated with miR-NC in the NEAT1WT group, while it was not affected in the NEAT1-MUT group (Fig. 4B). Moreover, the addition of miR-107 resulted in greater expression of NEAT1 enriched by Ago2 RIP in SH-SY5Y and SK-N-SH cells, whereas it showed little capacity for enrichment in the IgG RIP group (Fig. 4C and D). Then, the effect of NEAT1 on miR-107 expression was analyzed in SH-SY5Y and SK-N-SH cells. The results showed that overexpression of NEAT1 suppresses miR-107 levels in SH-SY5Y and SK-N-SH
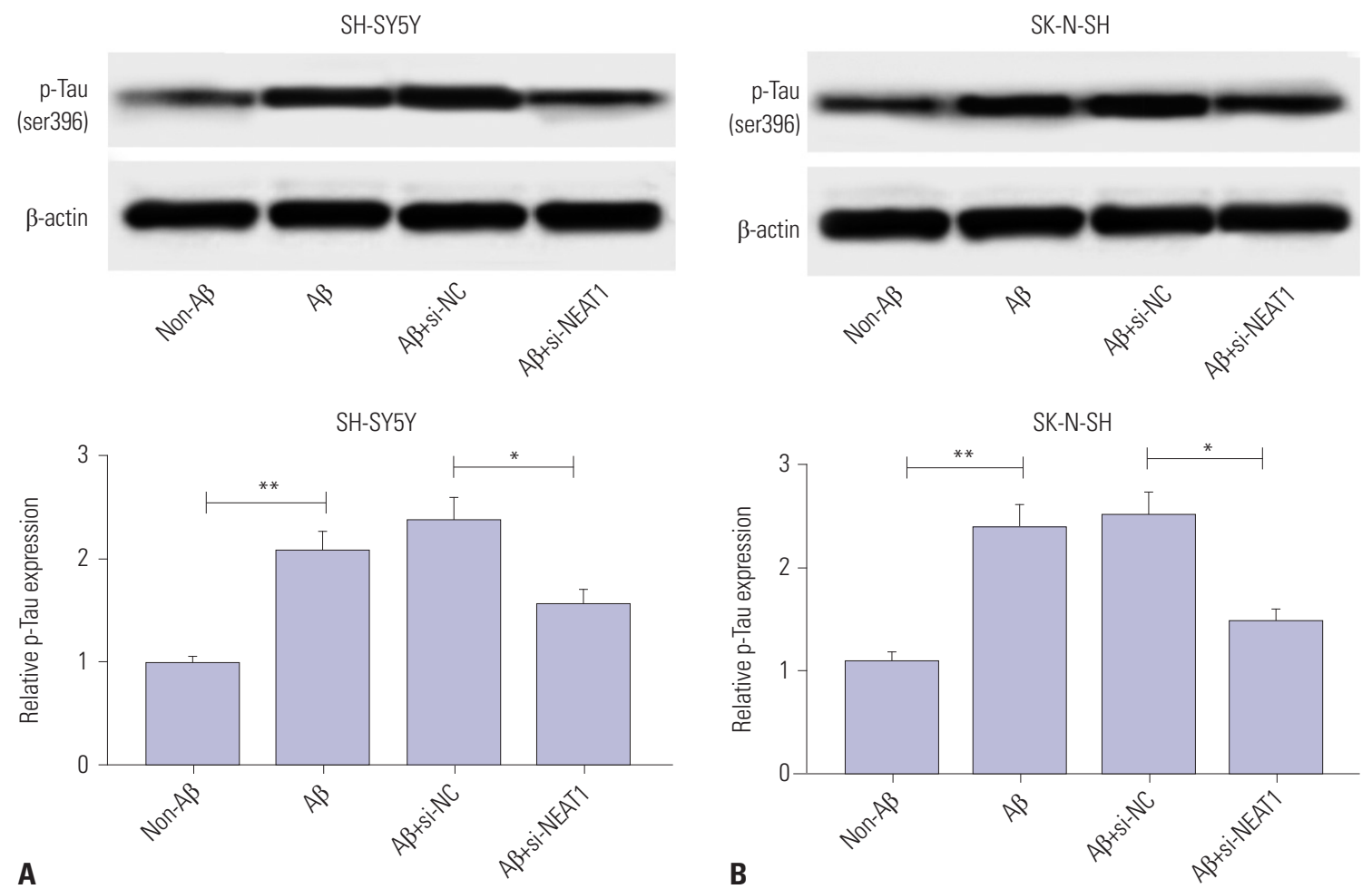

Fig. 3. Interference of NEAT1 weakens A $\beta$-induced phosphorylation of Tau in SH-SY5Y and SK-N-SH cells. The protein levels of phosphorylation of Tau (p-Tau) were measured in SH-SY5Y (A) and SK-N-SH (B) cells transfected with si-NEAT1 or si-NC after treatment of $10 \mu M A \beta$ for $24 \mathrm{~h}$ by Western blot. ${ }^{*} p<0.05,{ }^{* *} p<0.01$. NEAT1, nuclear enriched abundant transcript $1 ; A \beta$, amyloid $\beta_{1-42}$. 
Site: chr11:65191782-65191803[+]

NEAT1-WT 5 '... guaaagguuu-ucagAUGCUGCa...3'

$\operatorname{miR}-107$

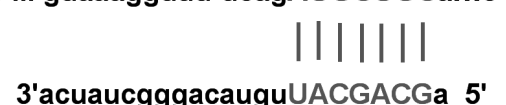

NEAT1-MUT 5'... guaaagguuu-ucagCGUAGUAa...3' A
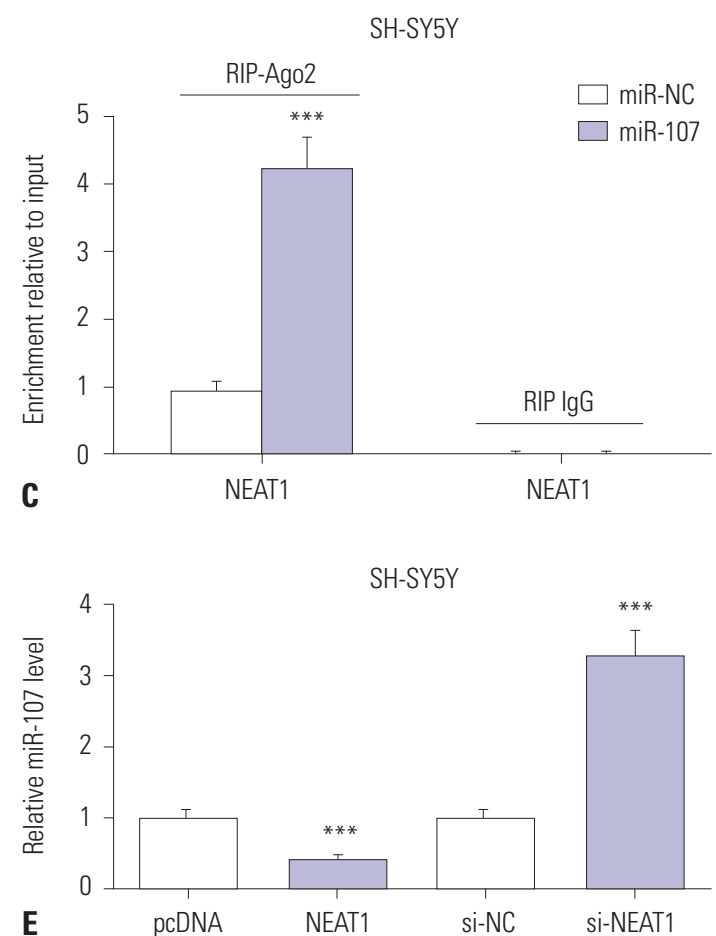

293T

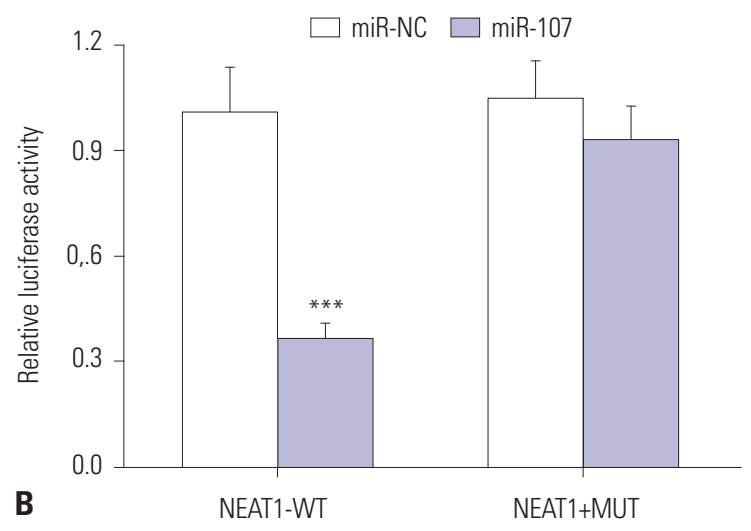

SK-N-SH
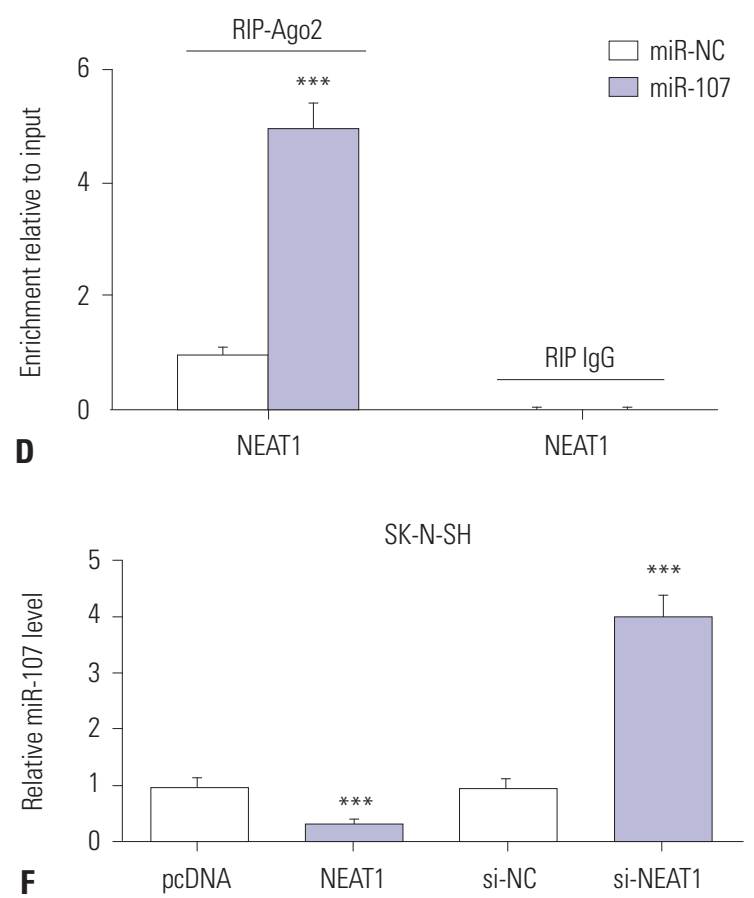

Fig. 4. miR-107 is bound to NEAT1. (A) The putative binding sites of NEAT1 and miR-107 were predicted by StarBase. (B) Luciferase activity was measured in 293T cells co-transfected with NEAT1-WT or NEAT1-MUT and miR-107 or miR-NC. (C and D) The enrichment of NEAT1 was detected in SHSY5Y and SK-N-SH cells transfected with miR-107 or miR-NC after RIP assay. (E and F) The expression of miR-107 was measured in SH-SY5Y and SK$\mathrm{N}-\mathrm{SH}$ cells transfected with pcDNA, NEAT1, si-NC or si-NEAT1 by qRT-PCR. ${ }^{* * *} p<0.001$. NEAT1, nuclear enriched abundant transcript 1.

cells, compared with pcDNA transfection, while its knockdown causes an opposite effect on miR-107 abundance (Fig. 4E and F). These results revealed that NEATl is a sponge of miR-107.

\section{MiR-107 is lowly expressed in Aß-treated SH-SY5Y and SK-N-SH cells}

Having established that miR-107 is targeted by NEAT1, the abundance of miR-107 was measured in SH-SY5Y and SK-NSH cells after treatment of $A \beta$. Compared with the non-treated group, treatment of $A \beta$ for $24 \mathrm{~h}$ led to a marked reduction in miR-107 expression in the two types of cells in a concentration dependent manner (Fig. 5A and B). Furthermore, a progressive decrease in miR-107 levels was observed in SH-SY5Y and
SK-N-SH cells after treatment of $10 \mu \mathrm{M} \mathrm{A} \beta$ in a time dependent manner (Fig. 5C and D). These data suggested that low expression of miR-107 might be associated with $\mathrm{AD}$ progression.

\section{NEAT1 mediates neuronal damage by sponging miR-} 107 in A $\beta$-treated SH-SY5Y and SK-N-SH cells

To analyze the potential role of miR-107 in AD progression, SH-SY5Y and SK-N-SH cells were transfected with miR-107 or miR-NC and then treated with $10 \mu \mathrm{M} A \beta$ for $24 \mathrm{~h}$. In result, the expression of miR-107 was effectively rescued by transfection of miR-107 mimic, compared with that in the miR-NC group, among A $\beta$-treated SH-SY5Y and SK-N-SH cells (Fig. 6A and B). Moreover, overexpression of miR-107 reversed A $\beta$-induced vi- 

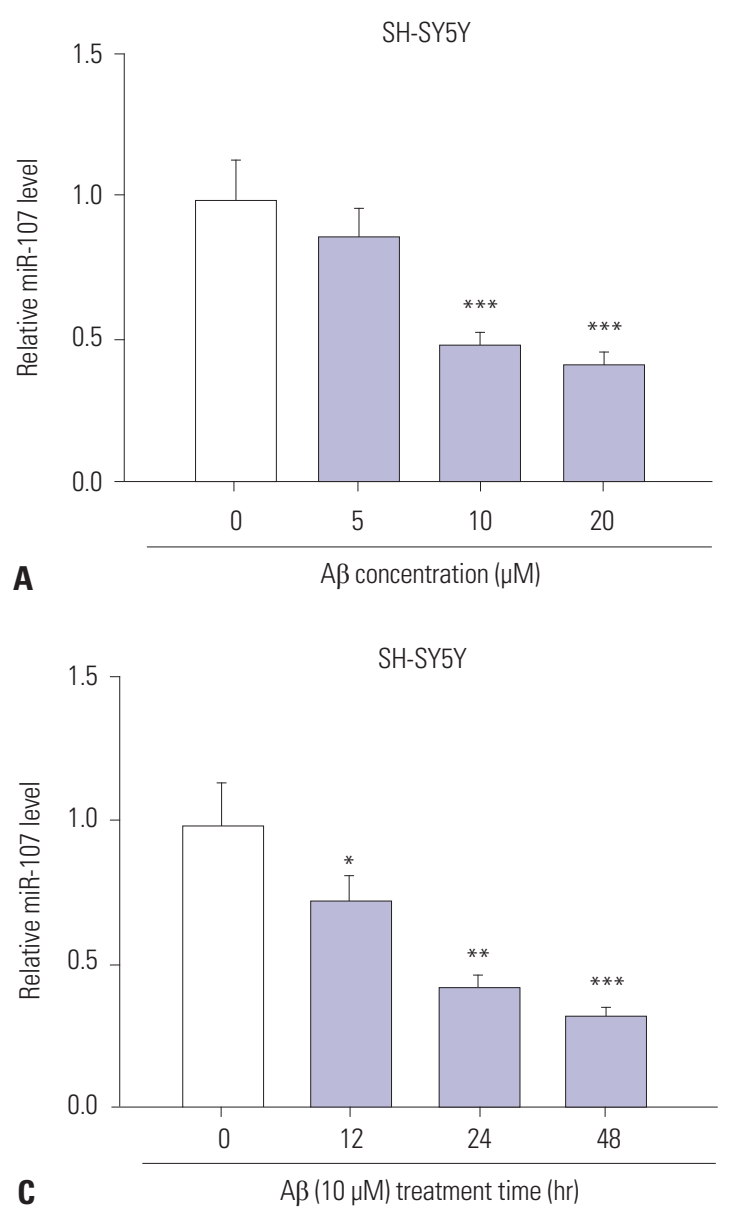
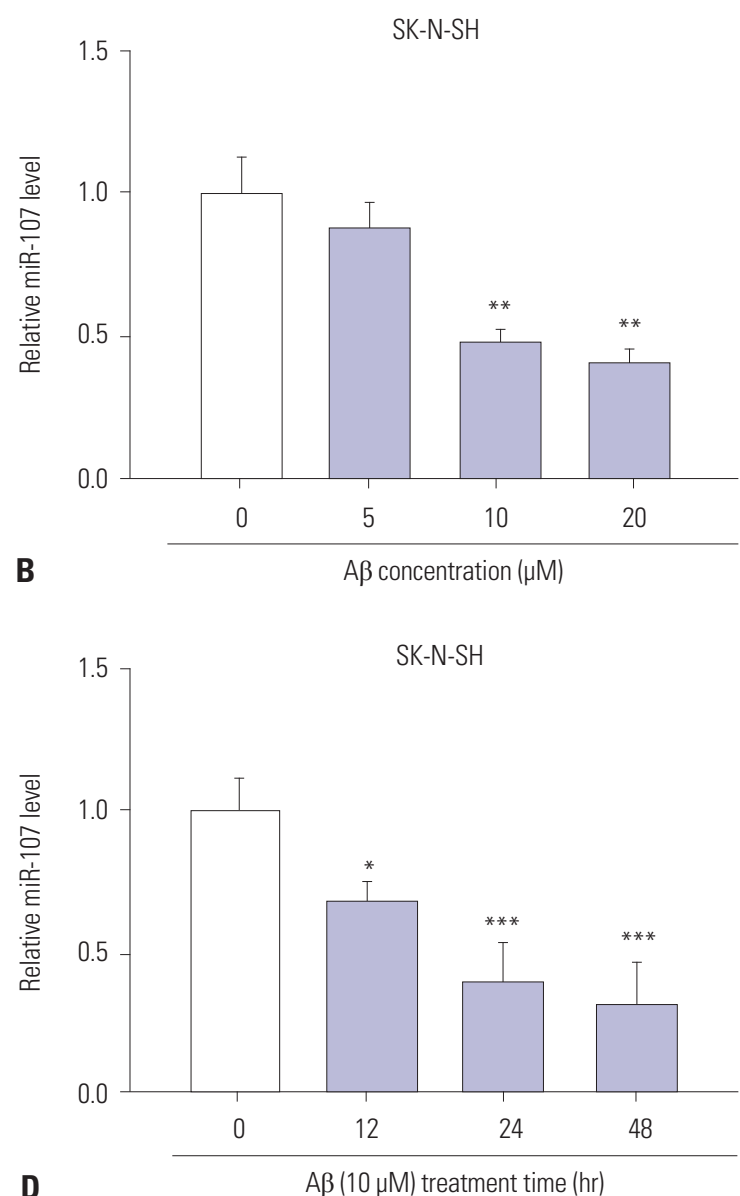

Fig. 5. The levels of miR-107 are decreased in Aß-treated SH-SY5Y and SK-N-SH cells. (A and B) The expression of miR-107 was measured in SHSY5Y and SK-N-SH cells after treatment of different concentrations of A $\beta$ for $24 \mathrm{~h}$ by qRT-PCR. (C and D) The abundance of miR-107 was detected in SH-SY5Y and SK-N-SH cells after treatment of $10 \mu \mathrm{M}$ A $\beta$ for different treatment times by qRT-PCR. ${ }^{*} p<0.05,{ }^{* *} p<0.01,{ }^{* * *} p<0.001$. A $\beta$, amyloid $\beta_{1-42}$.

ability inhibition and apoptosis induction in SH-SY5Y and SKN-SH cells (Fig. 6C-F). Meanwhile, knockdown of miR-107 aggravated $A \beta$-induced injury, while its knockdown alone showed little effect on cell viability and apoptosis in SH-SY5Y and SKN-SH cells without $A \beta$ treatment (Supplementary Fig. 1, only online). Furthermore, up-regulation of miR-107 abated A $\beta$ induced phosphorylation of Tau in SH-SY5Y and SK-N-SH cells, compared with miR-NC treatment (Fig. 7). These results highlighted the suppressive effect of miR-107 on A $\beta$-induced neuronal injury. Additionally, to explore whether miR-107 was associated with the regulatory effect of NEAT1 on AD development, SH-SY5Y and SK-N-SH cells were co-transfected with siNEAT1 and anti-miR-107 or anti-miR-NC before treatment with A $\beta$. qRT-PCR assay showed that the abundance of miR107 was obviously decreased in SH-SY5Y and SK-N-SH cells transfected with si-NEAT1 and anti-miR-107, compared with that in cells treated with si-NEATl and anti-miR-NC, after treatment of $A \beta$ (Fig. 6A and B). Additionally, exhaustion of miR107 alleviated the regulatory effect of NEAT1 knockdown on cell viability, apoptosis, and phosphorylation of Tau in $\mathrm{A} \beta$ treated SH-SY5Y and SK-N-SH cells (Figs. 6C-F and 7). These results indicated that NEAT1 knockdown mitigates $A \beta$ - induced neuronal damage by upregulating miR-107.

\section{DISCUSSION}

A $\beta$-treated SH-SY5Y and SK-N-SH cells have been widely used for establishment of $\mathrm{AD}$ models in vitro. ${ }^{19,20}$ In the present study, we also established an AD model in SH-SY5Y and SK-N$\mathrm{SH}$ cells via exposure of $\mathrm{A} \beta$. Our results showed that treatment of $A \beta$ leads to inhibition of cell viability and increases in apoptosis and p-Tau levels in SH-SY5Y and SK-N-SH cells, which supported the viability of our AD model. NEAT1, as a promising IncRNA, has been reported to contribute to neuronal injury in Huntington's disease and Parkinson's disease. ${ }^{9,11}$ On the basis of previous results, NEATl might play an important role in neurodegenerative disease. However, the role of NEAT1 in AD progression remains obscure. Here, we discovered that NEAT1 aggravates $\mathrm{A} \beta$-induced neuronal damage in $\mathrm{AD}$ by sponging miR-107.

Previous research suggested that NEAT1 is highly expressed in $\mathrm{AD}$ patients, compared to control brain tissues. ${ }^{12}$ Similarly, we also found high expression of NEAT1 in our A $\beta$-induced AD 

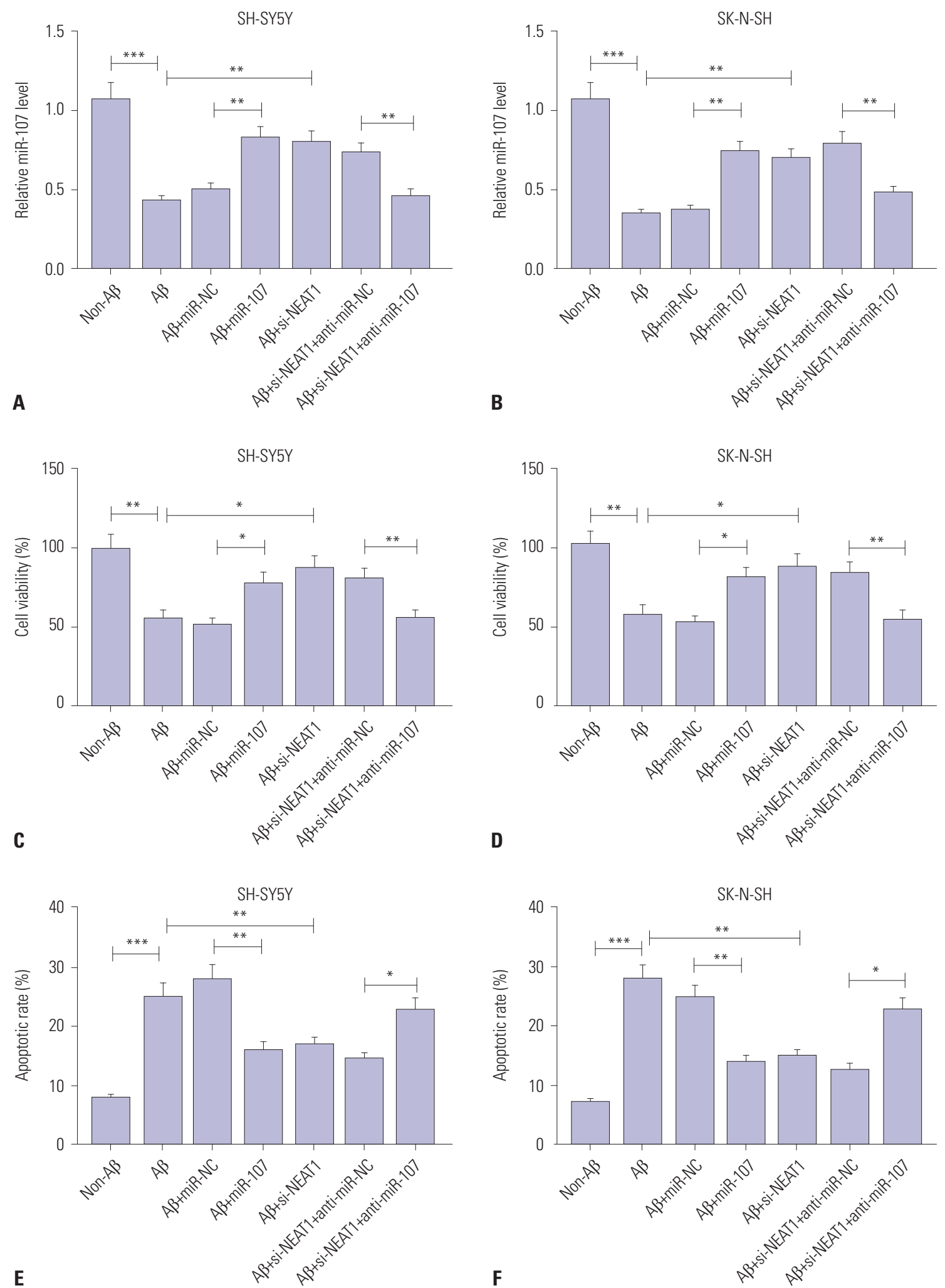

Fig. 6. Abrogation of miR-107 reverses the regulatory effect of NEAT1 knockdown on A $\beta$-induced neuronal damage in SH-SY5Y and SK-N-SH cells. (A and B) The expression of miR-107 was measured in SH-SY5Y and SK-N-SH cells transfected with miR-107, miR-NC, si-NEAT1, and anti-miR-NC or anti-miR-107 after treatment of $10 \mu \mathrm{M}$ A $\beta$ for $24 \mathrm{~h}$ by qRT-PCR. Cell viability (C and D) and apoptosis (E and F) were detected in SH-SY5Y and SK-N-SH cells transfected with miR-107, miR-NC, si-NEAT1, and anti-miR-NC or anti-miR-107 after treatment of $10 \mu \mathrm{M} A \beta$ for $24 \mathrm{~h}$ by MTT or flow cytometry. ${ }^{*} p<0.05,{ }^{* *} p<0.01,{ }^{* * *} p<0.001$. NEAT1, nuclear enriched abundant transcript $1 ; A \beta$, amyloid $\beta_{1-42}$. 

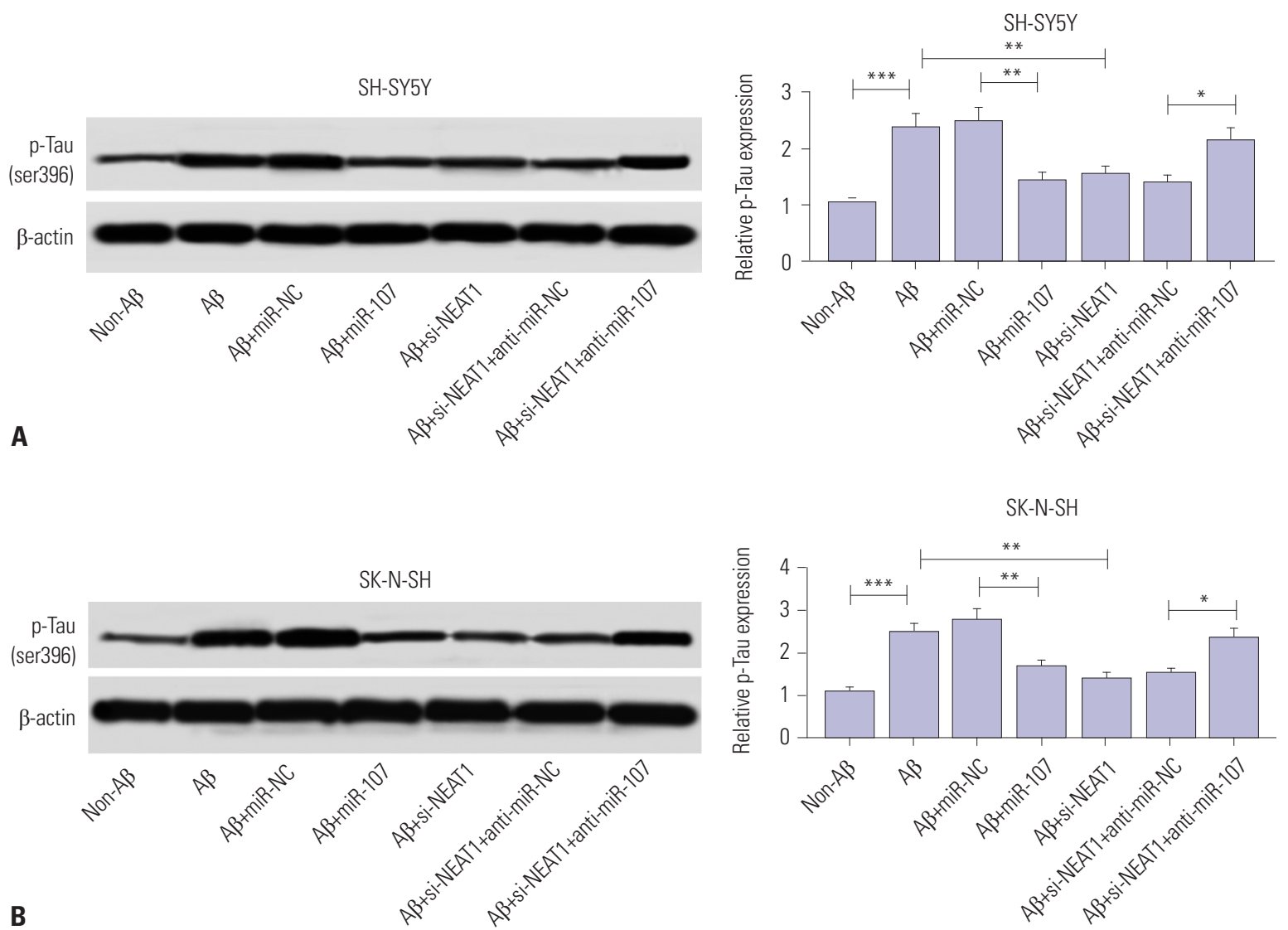

Fig. 7. miR-107 is involved in interference of NEAT1-mediated inhibition of phosphorylation of Tau (p-Tau) in A $\beta$-treated SH-SY5Y and SK-N-SH cells. The protein levels of $p$-Tau were detected in SH-SY5Y (A) and SK-N-SH (B) cells transfected with miR-107, miR-NC, si-NEAT1, and anti-miR-NC or antimiR-107 after treatment of $10 \mu \mathrm{M} \mathrm{A} \beta$ for $24 \mathrm{~h}$ by Western blot. ${ }^{*} p<0.05,{ }^{* *} p<0.01$, ${ }^{* * *} p<0.001$. NEAT1, nuclear enriched abundant transcript 1 ; $A \beta$, amyloid $\beta_{1-42 .}$

model in vitro. In this work, silencing of NEAT1 attenuated A $\beta$ induced inhibition of cell viability and increases in apoptosis and phosphorylation of Tau in vitro, indicating NEAT1 as a potential neuro-regulator in $\mathrm{AD}$ progression. Nevertheless, the underlying mechanism by which NEAT1 participates in neuronal damage in $\mathrm{AD}$ needs to be further explored. Former efforts have suggested NEAT1 as novel target for diagnosis and treatment of human cancers by functioning as a sponge of miRNAs. ${ }^{21}$ Chen, et al. ${ }^{22}$ reported that NEAT1 promotes ox-LDL-induced inflammatory and oxidative stress injury by sponging miR-128 in macrophages. Zhou, et al. ${ }^{23}$ suggested that abrogation of NEAT1 suppresses migration and invasion by regulating miR-132 in glioma cells. In this study, using luciferase activity and RIP assays, we confirmed the regulatory network of NEAT1 and miR-107 in SH-SY5Y and SK-N-SH cells, which was also demonstrated as a main pathway in progression of glioma and laryngeal squamous cell cancer. ${ }^{24-26}$

We discovered that miR-107 expression was reduced in our in vitro $A \beta$-induced $A D$ model, which is in agreement with previous works that reported low expression of miR-107 in $\mathrm{AD}$ brains. ${ }^{27,28} \mathrm{Liu}$, et al. ${ }^{29}$ reported that miR-107 prevented $\mathrm{A} \beta$ induced disruption of the blood-brain barrier (BBB) and dysfunction of endothelial cells in $\mathrm{AD}$ by regulating endophilin-1, suggesting that miR-107 might play a neuroprotective role in AD progression. Meanwhile, we hypothesized that NEAT1 could promote $\mathrm{BBB}$ dysfunction in $\mathrm{AD}$ by regulating miR-107, which warrants further study in the future. Moreover, Wang, et al. ${ }^{30}$ suggested that miR-107 was decreased in $\mathrm{AD}$ and that it might increase vulnerability to $\mathrm{AD}$. In this study, our results showed that addition of miR-107 inhibited $A \beta$-induced neuronal damage, which was consistent with research showing that miR-107 is negatively correlated with $A \beta$-induced reductions of cell viability and increases in apoptosis and phosphorylated Tau levels. ${ }^{31,32}$ These data demonstrate that miR-107 might be involved in a neuroprotective mechanism in $\mathrm{AD}$ progression. Meanwhile, we demonstrated that deficiency of miR107 counteracted interference of NEAT1-mediated inhibition of neuronal damage in $\mathrm{AD}$. This indicated that knockdown of NEAT1 plays a protective role in AD by regulating miR-107. Former efforts have suggested that rodent models are indispensable for research on $\mathrm{AD} .{ }^{33}$ Hence, an animal model of $\mathrm{AD}$ and clinical experiments should be performed in the future to investigate the role of NEAT1 in vivo. Moreover, the potential targets of miR-107 and promising signaling pathways should be explored in further studies for better understanding the mechanism. 
In conclusion, NEAT1 expression was increased in A $\beta$-treated SH-SY5Y and SK-N-SH cells. Knockdown of NEAT1 attenuated A $\beta$-induced neuronal injury, possibly via sponging miR-107, in an in vitro $\mathrm{A} \beta$-induced $\mathrm{AD}$ model, indicating a novel avenue for treatment of $\mathrm{AD}$.

\section{ACKNOWLEDGEMENTS}

This work was supported by City and College Strategic Cooperation Project of Nanchong (Grant No. 18SXHZ0576).

\section{AUTHOR CONTRIBUTIONS}

Conceptualization: Sha Ke, Fei Yang, Juan Tan, Bo Liao. Data curation: Juan Tan, Xiaoming Wang, Bo Liao, Fei Yang, Zhaohui Yang. Formal analysis: Sha Ke, Fei Yang, Juan Tan, Bo Liao. Funding acquisition: Sha Ke, Juan Tan, Xiaoming Wang, Bo Liao. Investigation: Bo Liao, Fei Yang. Methodology: Sha Ke, Bo Liao, Fei Yang. Project administration: Juan Tan, Fei Yang. Resources: Sha Ke, Bo Liao. Software: Sha Ke, Juan Tan. Supervision: Sha Ke, Fei Yang. Validation: Fei Yang, Bo Liao. Visualization: Sha Ke, Juan Tan. Writing-original draft: Sha Ke, Bo Liao, Fei Yang, Xiaoming Wang. Writing-review \& editing: Sha Ke, Zhaohui Yang, Bo Liao.

\section{ORCID iDs}

Sha Ke Zhaohui Yang Fei Yang Xiaoming Wang Juan Tan Bo Liao https://orcid.org/0000-0002-8945-9437 https://orcid.org/0000-0002-6400-3900 https://orcid.org/0000-0002-4460-3132 https://orcid.org/0000-0002-5640-9572 https://orcid.org/0000-0003-3770-918X https://orcid.org/0000-0002-6596-2302

\section{REFERENCES}

1. Scheltens P, Blennow K, Breteler MM, de Strooper B, Frisoni GB, Salloway S, et al. Alzheimer's disease. Lancet 2016;388:505-17.

2. Chen Y, Fu AKY, Ip NY. Synaptic dysfunction in Alzheimer's disease: mechanisms and therapeutic strategies. Pharmacol Ther 2019;195:186-98.

3. Nasica-Labouze J, Nguyen PH, Sterpone F, Berthoumieu O, Buchete NV, Coté S, et al. Amyloid $\beta$ protein and Alzheimer's disease: when computer simulations complement experimental studies. Chem Rev 2015;115:3518-63.

4. Li C, Götz J. Tau-based therapies in neurodegeneration: opportunities and challenges. Nat Rev Drug Discov 2017;16:863-83.

5. Idda ML, Munk R, Abdelmohsen K, Gorospe M. Noncoding RNAs in Alzheimer's disease. Wiley Interdiscip Rev RNA 2018;9;e1463.

6. Wang DQ, Fu P, Yao C, Zhu LS, Hou TY, Chen JG, et al. Long noncoding RNAs, novel culprits, or bodyguards in neurodegenerative diseases. Mol Ther Nucleic Acids 2018;10:269-76.

7. Zhang L, Fang Y, Cheng X, Lian YJ, Xu HL. Silencing of long noncoding RNA SOX21-AS1 relieves neuronal oxidative stress injury in mice with Alzheimer's disease by upregulating FZD3/5 via the Wnt signaling pathway. Mol Neurobiol 2019;56:3522-37.

8. Gu C, Chen C, Wu R, Dong T, Hu X, Yao Y, et al. Long noncoding RNA EBF3-AS promotes neuron apoptosis in Alzheimer's disease. DNA Cell Biol 2018;37:220-6.

9. Chanda K, Das S, Chakraborty J, Bucha S, Maitra A, Chatterjee R, et al. Altered levels of long ncRNAs Meg3 and Neat1 in cell and animal models of Huntington's disease. RNA Biol 2018;15:1348-63.

10. Yan W, Chen ZY, Chen JQ, Chen HM. LncRNA NEAT1 promotes autophagy in MPTP-induced Parkinson's disease through stabilizing PINK1 protein. Biochem Biophys Res Commun 2018;496: 1019-24.

11. Liu Y, Lu Z. Long non-coding RNA NEAT1 mediates the toxic of Parkinson's disease induced by MPTP/MPP+ via regulation of gene expression. Clin Exp Pharmacol Physiol 2018;45:841-8.

12. Spreafico M, Grillo B, Rusconi F, Battaglioli E, Venturin M. Multiple layers of CDK5R1 regulation in Alzheimer's disease implicate long non-coding RNAs. Int J Mol Sci 2018;19:2022.

13. Martinez B, Peplow PV. MicroRNAs as diagnostic and therapeutic tools for Alzheimer's disease: advances and limitations. Neural Regen Res 2019;14:242-55.

14. Finnerty JR, Wang WX, Hébert SS, Wilfred BR, Mao G, Nelson PT. The miR-15/107 group of microRNA genes: evolutionary biology, cellular functions, and roles in human diseases. J Mol Biol 2010; 402:491-509.

15. Foley NH, O'Neill LA. miR-107: a toll-like receptor-regulated miRNA dysregulated in obesity and type II diabetes. J Leukoc Biol 2012; 92:521-7.

16. Jiang ZP, Zhou TB. Role of miR-107 and its signaling pathways in diseases. J Recept Signal Transduct Res 2014;34:338-41.

17. Fransquet PD, Ryan J. Micro RNA as a potential blood-based epigenetic biomarker for Alzheimer's disease. Clin Biochem 2018; 58:5-14.

18. Livak KJ, Schmittgen TD. Analysis of relative gene expression data using real-time quantitative PCR and the $2^{\Delta \triangle C T}$ Method. Methods 2001;25:402-8.

19. Jiang Y, Xu B, Chen J, Sui Y, Ren L, Li J, et al. Micro-RNA-137 inhibits tau hyperphosphorylation in Alzheimer's disease and targets the CACNA1C gene in transgenic mice and human neuroblastoma SH-SY5Y cells. Med Sci Monit 2018;24:5635-44.

20. Li Q, Li X, Wang L, Zhang Y, Chen L. miR-98-5p acts as a target for Alzheimer's disease by regulating $A \beta$ production through modulating SNX6 expression. J Mol Neurosci 2016;60:413-20.

21. Dong P, Xiong Y, Yue J, Hanley SJB, Kobayashi N, Todo Y, et al. Long non-coding RNA NEAT1: a novel target for diagnosis and therapy in human tumors. Front Genet 2018;9:471.

22. Chen DD, Hui LL, Zhang XC, Chang Q. NEAT1 contributes to oxLDL-induced inflammation and oxidative stress in macrophages through inhibiting miR-128. J Cell Biochem 2018;120:2493-501.

23. Zhou K, Zhang C, Yao H, Zhang X, Zhou Y, Che Y, et al. Knockdown of long non-coding RNA NEAT1 inhibits glioma cell migration and invasion via modulation of SOX2 targeted by miR- 132 . Mol Cancer 2018;17:105.

24. Zhen Y, Nan Y, Guo S, Zhang L, Li G, Yue S, et al. Knockdown of NEAT1 repressed the malignant progression of glioma through sponging miR-107 and inhibiting CDK14. J Cell Physiol 2019;234: 10671-9.

25. Yang X, Xiao Z, Du X, Huang L, Du G. Silencing of the long noncoding RNA NEAT1 suppresses glioma stem-like properties through modulation of the miR-107/CDK6 pathway. Oncol Rep 2017;37: $555-62$.

26. Wang P, Wu T, Zhou H, Jin Q, He G, Yu H, et al. Long noncoding RNA NEAT1 promotes laryngeal squamous cell cancer through regulating miR-107/CDK6 pathway. J Exp Clin Cancer Res 2016; 35:22.

27. Gupta P, Bhattacharjee S, Sharma AR, Sharma G, Lee SS, Chakraborty C. miRNAs in Alzheimer disease - a therapeutic perspective. Curr Alzheimer Res 2017;14:1198-206.

28. Moncini S, Lunghi M, Valmadre A, Grasso M, Del Vescovo V, Riva 
P, et al. The miR-15/107 family of microRNA genes regulates CD$\mathrm{K} 5 \mathrm{R} 1 / \mathrm{p} 35$ with implications for Alzheimer's disease pathogenesis. Mol Neurobiol 2017;54:4329-42.

29. Liu W, Cai H, Lin M, Zhu L, Gao L, Zhong R, et al. MicroRNA-107 prevents amyloid-beta induced blood-brain barrier disruption and endothelial cell dysfunction by targeting Endophilin-1. Exp Cell Res 2016;343:248-57.

30. Wang WX, Rajeev BW, Stromberg AJ, Ren N, Tang G, Huang Q, et al. The expression of microRNA miR-107 decreases early in $\mathrm{Al}$ zheimer's disease and may accelerate disease progression through regulation of beta-site amyloid precursor protein-cleaving enzyme 1. J Neurosci 2008;28:1213-23.

31. Jiao Y, Kong L, Yao Y, Li S, Tao Z, Yan Y, et al. Osthole decreases beta amyloid levels through up-regulation of miR-107 in Alzheimer's disease. Neuropharmacology 2016;108:332-44.

32. Shu B, Zhang X, Du G, Fu Q, Huang L. MicroRNA-107 prevents amyloid- $\beta$-induced neurotoxicity and memory impairment in mice. Int J Mol Med 2018;41:1665-72.

33. Götz J, Bodea LG, Goedert M. Rodent models for Alzheimer disease. Nat Rev Neurosci 2018;19:583-98. 